\title{
Erratum to: An improved block-based matching algorithm of copy-move forgery detection
}

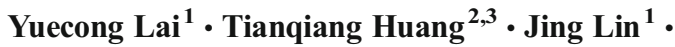 \\ Henan $\mathrm{Lu}^{1}$
}

Published online: 6 September 2017

(C) Springer Science+Business Media, LLC 2017

\section{Erratum to: Multimed Tools Appl \\ https://doi.org/10.1007/s11042-017-5094-y}

In the original publication, reference citations in Fig. 4 were incorrectly written as [5], [6], [7], [10] and [11]. It should be written as [14], [9], [11], [7] and [6]. The original article was corrected.

The online version of the original article can be found at https://doi.org/10.1007/s11042-017-5094-y

\section{Tianqiang Huang}

fjhtq@fjnu.edu.cn

1 School of Mathematics and Computer Science, Fujian Normal University, Fuzhou 350007, China

2 Faculty of Software, Fujian Normal University, Fuzhou 350007, China

3 Fujian Engineering Research Center of Public Service Big Data Mining and Application, Fujian Normal University, Fuzhou 350007, China 